\title{
On the representation number of a crown graph
}

\author{
Marc Glen ${ }^{1}$, Sergey Kitaev ${ }^{1}$, and Artem Pyatkin*2 \\ ${ }^{1}$ Department of Computer and Information Sciences, University of Strathclyde, \\ 26 Richmond Street, Glasgow G1 1XH, UK \\ ${ }^{2}$ Sobolev Institute of Mathematics, Novosibirsk State University, 630090 \\ Novosibirsk, Russia
}

\begin{abstract}
A graph $G=(V, E)$ is word-representable if there exists a word $w$ over the alphabet $V$ such that letters $x$ and $y$ alternate in $w$ if and only if $x y$ is an edge in $E$. It is known that any word-representable graph $G$ is $k$-word-representable for some $k$, that is, there exists a word $w$ representing $G$ such that each letter occurs exactly $k$ times in $w$. The minimum such $k$ is called $G$ 's representation number.

A crown graph (also known as a cocktail party graph) $H_{n, n}$ is a graph obtained from the complete bipartite graph $K_{n, n}$ by removing a perfect matching. In this paper we show that for $n \geq 5, H_{n, n}$ 's representation number is $\lceil n / 2\rceil$. This result not only provides a complete solution to the open Problem 7.4.2 in [8], but also gives a negative answer to the question raised in Problem 7.2.7 in [8] on 3-wordrepresentability of bipartite graphs. As a byproduct we obtain a new example of a graph class with a high representation number.
\end{abstract}

Keywords: word-representable graph, crown graph, cocktail party graph, representation number

*This research was partially supported by RFBR (project 15-01-00976). 


\section{Introduction}

Suppose that $w$ is a word over some alphabet and $x$ and $y$ are two distinct letters in $w$. We say that $x$ and $y$ alternate in $w$ if after deleting in $w$ all letters but the copies of $x$ and $y$ we either obtain a word $x y x y \cdots$ (of even or odd length) or a word yxyx $\cdots$ (of even or odd length).

A graph $G=(V, E)$ is word-representable if there exists a word $w$ over the alphabet $V$ such that letters $x$ and $y$ alternate in $w$ if and only if $x y$ is an edge in $E$. For example, the cycle graph on 4 vertices labeled by 1,2, 3 and 4 in clockwise direction can be represented by the word 14213243 .

There is a long line of research on word-representable graphs, which is summarised in the recently published book [8]. The roots of the theory of word-representable graphs are in the study of the celebrated Perkins semigroup $[10,13]$ which has played a central role in semigroup theory since 1960, particularly as a source of examples and counterexamples. However, the most interesting aspect of word-representable graphs from an algebraic point of view seems to be the notion of a semi-transitive orientation [6], which generalizes partial orders. It was shown in [6] that a graph is word-representable if and only if it admits a semi-transitive orientation.

More motivation points to study word representable graphs include the fact exposed in [8] that these graphs generalize several important classes of graphs such as circle graphs [4], 3-colourable graphs [1] and comparability graphs [12]. Relevance of word-representable graphs to scheduling problems was explained in [6] and it was based on [5]. Furthermore, the study of word-representable graphs is interesting from an algorithmic point of view as explained in [8]. For example, the Maximum Clique problem is polynomially solvable on word-representable graphs [8] while this problem is generally NP-complete [3]. Finally, word-representable graphs is an important class among other graph classes considered in the literature that are defined using words. Examples of other such classes of graphs are polygon-circle graphs [11] and word-digraphs [2].

It was shown in [9] that if a graph $G$ is word-representable then it is $k$-word-representable for some $k$, that is, $G$ can be represented by a $k$-uniform word $w$, i. e. a word containing $k$ copies of each letter. In such a context we say that $w$ k-represents $G$. For example, the cycle graph on 4 vertices mentioned above can be 2-represented 
by the word 14213243. Thus, when discussing word-representability, one need only consider $k$-uniform words. The nice property of such words is that any cyclic shift of a $k$-uniform word represents the same graph [9]. The minimum $k$ for which a word-representable graph $G$ is $k$-word-representable is called $G$ 's representation number.

The following observation trivially follows from the definitions.

Observation 1. The class of complete graphs coincides with the class of 1-word-representable graphs. In particular, the complete graph's representation number is 1 .

\subsection{Representation of crown graphs}

A crown graph (also known as a cocktail party graph) $H_{n, n}$ is a graph obtained from the complete bipartite graph $K_{n, n}$ by removing a perfect matching. Formally, $V\left(H_{n, n}\right)=\left\{1, \ldots, n, 1^{\prime}, \ldots, n^{\prime}\right\}$ and $E\left(H_{n, n}\right)=$ $\left\{i j^{\prime} \mid i \neq j\right\}$. First four examples of such graphs are presented in Figure 1.

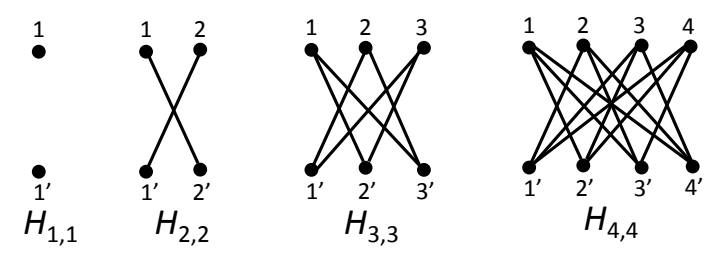

Figure 1: The crown graph $H_{n, n}$ for $n=1,2,3,4$

Crown graphs are of special importance in the theory of wordrepresentable graphs. More precisely, they appear in the construction of graphs requiring long words representing them [6]. Note that these graphs also appear in the theory of partially ordered sets as those defining partial orders that require many linear orders to be represented.

Each crown graph, being a bipartite graph, is a comparability graph (that is, a transitively orientable graph), and thus it can be represented by a concatenation of permutations [10]. Moreover, it follows from [6], and also is discussed in Section 7.4 in [8], that $H_{n, n}$ can be represented as a concatenation of $n$ permutations but it cannot be represented as a concatenation of a fewer permutations. These results on crown graphs were obtained by exploiting the idea of representation of a poset as an intersection of several linear orders. Thus, the representation number 


\begin{tabular}{c|c}
$n$ & representation of $H_{n, n}$ by concatenation of $n$ permutations \\
\hline 1 & $11^{\prime} 1^{\prime} 1$ \\
\hline 2 & $12^{\prime} 21^{\prime} 21^{\prime} 12^{\prime}$ \\
\hline 3 & $123^{\prime} 32^{\prime} 1^{\prime} 132^{\prime} 23^{\prime} 1^{\prime} 231^{\prime} 13^{\prime} 2^{\prime}$ \\
\hline 4 & $1234^{\prime} 43^{\prime} 2^{\prime} 1^{\prime} 1243^{\prime} 34^{\prime} 2^{\prime} 1^{\prime} 1342^{\prime} 24^{\prime} 3^{\prime} 1^{\prime} 2341^{\prime} 14^{\prime} 3^{\prime} 2^{\prime}$ \\
\hline
\end{tabular}

Table 1: Representing $H_{n, n}$ as a concatenation of $n$ permutations

of $H_{n, n}$ is at most $n$. See Table 1 (appearing in [8]) for the words representing the graphs in Figure 1 as concatenation of permutations.

It was noticed in [7] that, for example, $H_{3,3}$ can be represented using two copies of each letter as $3^{\prime} 32^{\prime} 1^{\prime} 132^{\prime} 23^{\prime} 1^{\prime} 231^{\prime} 1$ (as opposed to three copies used in Table 1 to represent it) if we drop the requirement to represent crown graphs as concatenation of permutations. On the other hand, $H_{4,4}$ is the three-dimensional cube, which is the prism graph $\mathrm{Pr}_{4}$, so that $H_{4,4}$ is 3-word-representable by Proposition 15 in [9], while four copies of each letter are used in Table 1 to represent this graph. Note that $H_{4,4}$ is not 2-word-representable by Theorem 18 in [7].

These observations led to Problem 7.4.2 on page 172 in [8] essentially asking to find the representation number of a crown graph $H_{n, n}$. A relevant Problem 7.2.7 on page 169 in [8] asks whether each bipartite graph is 3 -word-representable. When we started to investigate these problems, we established that both $H_{5,5}$ and $H_{6,6}$ are 3 -word-representable, which not only suggested that the representation number of a crown graph could be the constant 3, but also that any bipartite graph could be 3 -word-representable since crown graphs seem to be the most difficult among them to be represented.

In this paper we completely solve the former problem (Problem 7.4.2) and provide the negative answer to the question in the latter problem (Problem 7.2.7) by showing that if $n \geq 5$ then the crown graph $H_{n, n}$, being a bipartite graph, is $\lceil n / 2\rceil$-representable (see Theorem 5). Thus, crown graphs are another example of a graph class with high representation number. Note that non-bipartite graphs obtained from crown graphs by adding an all-adjacent vertex (i.e. apex) require roughly twice as long words representing them (see Section 4.2.1 in $[8])$. 


\subsection{Organization of the paper and some defi- nitions}

The paper is organized as follows. In Section 2, we find a lower bound for the representation number of $H_{n, n}$, while in Section 3 we provide a construction of words representing $H_{n, n}$ that match our lower bound. Finally, in Section 4 we provide some concluding remarks including directions for further research.

We conclude the introduction with a number of technical definitions to be used in the paper.

A factor of a word is a number of consecutive letters in the word. For example, the set of all factors of the word 1132 of length at most 2 is $\{1,2,3,11,13,32\}$. A subword of a word is a subsequence of letters in the word. For instance, 56, 5212 and 361 are examples of subwords in 3526162. The subword of a word $w$ induced by a set $A$ is obtained by removing all elements in $w$ not belonging to $A$. For example, if $A=\{2,4,5\}$ then the subword of 223141565 induced by $A$ is 22455 .

For a vertex $v$ in a graph $G$ denote by $N(v)$ the neighbourhood of $v$, i.e. the set of vertices adjacent to $v$. Clearly, if a graph is bipartite then the neighbourhood of each vertex induces an independent set.

\section{A lower bound for the representa- tion number of $H_{n, n}$}

For a word $w$, let $l(w)$ and $r(w)$ be its first and last letters, respectively.

Let $w$ be a word that $k$-represents a graph $G=(V, E)$. A subset $A \subseteq V$ is splittable if there is a cyclic shift of the word $w$ such that the subword induced by the set $A$ has the form $P_{1} \cdots P_{k}$ where each $P_{i}$ is a permutation of $A$. For a splittable set $A$, a canonical shift of $w$, with respect to $A$, is a cyclic shift of $w$ that puts $l\left(P_{1}\right)$ at the beginning of the word. Note that up to renaming permutations there is a unique canonical shift. The following proposition gives an example of a splittable set.

Proposition 1. For any vertex $v \in V$ in a word-representable graph $G=(V, E)$, the set $A=N(v)$ is splittable.

Proof. Consider a cyclic shift of a word $w k$-representing $G$ that puts $v$ at the beginning of the word. Then between any two occurrences of $v$ (and after the last one) each letter from $A$ occurs exactly once, i.e. 
the subword induced by $A$ is a concatenation of permutations. Note, however, that this shift is not canonical with respect to $A$.

For a letter $x$, denote by $x_{i}$ its $i$-th occurrence in a word $w$ (from left to right). We write $x_{i}<y_{j}$ if the $i$-th occurrence of $x$ is to the left of the $j$-th occurrence of $y$ in $w$. Clearly, if $A$ is splittable, then for every $a, b \in A$ and for all $i, j$ such that $1 \leq i<j \leq k$, we have $a_{i}<b_{j}$.

Lemma 2. Let a word $w$-represent a graph $G=(V, E)$ and $A \subset V$ be a splittable set. Further, let $a, b \in A, x \notin A$ and $a x, b x \in E$. If in a canonical shift of $w a_{1}<x_{1}<b_{1}$ then $a b \in E$.

Proof. Let $a_{1}<x_{1}<b_{1}$. Since $A$ is splittable, $b_{i}<a_{i+1}$ for each $i$. Since both $a$ and $b$ are adjacent to $x$, we have $a_{i}<x_{i}<b_{i}$ for every $i=1, \ldots, k$. Therefore, $a$ and $b$ alternate in $w$ and must be adjacent in $G$.

Lemma 3. If $n \geq 5$ then in any word $w k$-representing $H_{n, n}$ the set $A=\{1, \ldots, n\}$ is splittable.

Proof. By Proposition 1, the set $B:=N\left(1^{\prime}\right)=\{2, \ldots, n\}$ is splittable, i.e. there is a cyclic shift of $w$ in which the letters of $B$ form the subword $P_{1}^{\prime} \cdots P_{k}^{\prime}$, where $P_{i}^{\prime}$ is a permutation of $B$. Let a canonical shift of $w$ with respect to $B$ be $P_{1} I_{1} \ldots P_{k} I_{k}$, where for $i=1, \ldots, k$, the factor $P_{i}$ begins at $l\left(P_{i}^{\prime}\right)$ and ends at $r\left(P_{i}^{\prime}\right)$, and $I_{i}$ s are (possibly empty) factors lying between $r\left(P_{i}^{\prime}\right)$ and $l\left(P_{i+1}^{\prime}\right)$. We begin by proving the following fact.

Claim 1. For every $t \geq 1$ and $i \geq 1$ such that $i+t-1 \leq k$, the factor $U=P_{i} I_{i} \cdots I_{i+t-2} P_{i+t-1}$ of $w$ contains at most $t$ copies of the letter 1 .

Proof of Claim 1. Indeed, suppose not. Using a cyclic shift of $w$ if necessary, without loss of generality we can assume that in a problematic case $i=1$. First consider the case $t=1$. That is, we assume that $P_{1}$ contains at least two 1 s. Let $a=l\left(P_{1}\right), b=r\left(P_{1}\right)$ and $x \in V \backslash\left\{1, a, b, 1^{\prime}, 2^{\prime}, \ldots, n^{\prime}\right\}$. Recall that $a, b$ belong to the splittable set $B$. Then the letter $x^{\prime}$ occurs exactly once between any two consecutive occurrences of 1 , in particular, between the first two occurrences. Hence we have $a_{1}<x_{1}^{\prime}<b_{1}$. Since both $a$ and $b$ are adjacent to $x^{\prime}$, it follows from Lemma 2 that $a b \in E$, contradiction. 
Now let $t \geq 2$. Let $a=l\left(P_{1}\right), b=r\left(P_{1}\right), c=r\left(P_{t}\right)$ and $x \in$ $V \backslash\left\{1, a, b, c, 1^{\prime}, 2^{\prime}, \ldots, n^{\prime}\right\}$ (recall that $n \geq 5$ ), and suppose that there are at least $t+1$ occurrences of 1 between $a$ and $c$. Note that $a \neq b$, but it is possible that $a=c$ or $a=b$. Since $1 x^{\prime} \in E$, there must be at least $t$ occurrences of $x^{\prime}$ between $a$ and $c$. By Lemma 2, no $x^{\prime}$ can appear between $a_{1}$ and $b_{1}$. However, $c$ appears exactly once between $a_{1}$ and $b_{1}$ (possibly coinciding with one of them) because $P_{1}$ contains the permutation $P_{1}^{\prime}$ over $B$ as a subword. Moreover, there are exactly $t$ occurrences of $c$ in $U$. Therefore, the subword of $U$ induced by $c$ and $x^{\prime}$ starts and ends with $c$ and contains at least $t$ copies of $x^{\prime}$. Clearly, such subword cannot be alternating, which contradicts $c x^{\prime} \in E$. Claim 1 is proved.

It follows from Claim 1 that each $P_{i} I_{i}$ contains at most two $1 \mathrm{~s}$, since $P_{i} I_{i} P_{i+1}$ contains at most two $1 \mathrm{~s}$ for $1 \leq i \leq k-1$. If each of $P_{i} I_{i}$ contains exactly one 1 then add 1 to each $P_{i}^{\prime}$ to obtain the concatenation of permutations for the set $A$ showing that it is splittable. Otherwise, some $P_{i} I_{i}$ must contain at least two 1s. Without loss of generality, $i=1$ (otherwise, we can apply a cyclic shift and rename the permutations). By Claim 1 applied to $P_{1}$ and $P_{1} I_{1} P_{2}$, at least one of $1 \mathrm{~s}$ must be in $I_{1}$ and $P_{2}$ contains no $1 \mathrm{~s}$. So, add the first occurrence of 1 to $P_{1}^{\prime}$ and the second one to $P_{2}^{\prime}$. If $I_{2}$ contains no 1 s we apply the same arguments to the word obtained from $w$ by removing the factor $P_{1} I_{1} P_{2} I_{2}$. Otherwise, again by Claim 1 applied to $P_{1} I_{1} P_{2} I_{2} P_{3}$, $I_{2}$ has one $1, P_{3}$ has no 1 and we add this 1 to $P_{3}^{\prime}$ and continue in the same way showing that $w$ contains as a subword a concatenation of permutations over $A$, and thus $A$ is splittable.

Lemma 4. Let $n \geq 5$ and $w$-represents $H_{n, n}$. Also, let $P_{1}^{\prime} \cdots P_{k}^{\prime}$ be a subword of (a cyclic shift of) $w$ that is a concatenation of permutations over $A=\{1, \ldots, n\}$ (existing by Lemma 3). Then for every a $\in A$ there is $j \in\{1, \ldots, k\}$ such that $a=l\left(P_{j}^{\prime}\right)$ or $a=r\left(P_{j}^{\prime}\right)$.

Proof. Assume that the letter 1 is never the first or the last letter of any permutation $P_{j}^{\prime}$. Consider a canonical shift of $w$ for the set $A$ and define the subwords $P_{i}$ and $I_{i}$ for permutations $P_{i}^{\prime}$ in the same way as in the proof of Lemma 3. Since $l\left(P_{1}\right) \neq 1$ and $r\left(P_{1}\right) \neq 1$, no $1^{\prime}$ can appear between $l\left(P_{1}\right)$ and $r\left(P_{1}\right)$ by Lemma 2 . This is true for any $P_{i}$ since we can apply a cyclic shift and rename $P_{i}$ and $P_{1}$. Moreover, no $I_{i}$ can have two or more $1^{\prime} \mathrm{s}$, or no $1^{\prime} \mathrm{s}$ at all, because otherwise $1^{\prime}$ would not be adjacent to the vertices in $\{2, \ldots, n\}$. 
But since each $P_{j}$ for $j=1, \ldots, k$ contains one 1 , the letters 1 and $1^{\prime}$ alternate in $w$, i.e. the vertices 1 and $1^{\prime}$ must be adjacent, contradicting the definition of $H_{n, n}$.

Theorem 5. For $n \geq 1$, the representation number of $H_{n, n}$ is at least $\lceil n / 2\rceil$.

Proof. We consider three cases.

- The statement is trivial for $n=1,2$ since each graph requires at least one copy of each letter to be represented.

- None of $H_{n, n}$ 's is a complete graph, and thus, by Observation 1 , the statement is true for $n=3,4$.

- Let $n \geq 5$. Since the set $A=\{1, \ldots, n\}$ is splittable by Lemma 3 , and each of its $n$ letters must be the first or the last letter of some permutation $P_{j}^{\prime}$ for $j=1, \ldots, k$ by Lemma 4 , we have the inequality $2 k \geq n$. Since $k$ is an integer, we obtain the bound $k \geq\lceil n / 2\rceil$.

\section{An upper bound for the representa- tion number of $H_{n, n}$}

In this section we provide a construction that shows that the bound in Theorem 5 is tight for all $n$ except $n=1,2,4$. We need the following auxiliary fact.

Lemma 6. If $n=2 k \geq 6$ then for every partition of the set $A=$ $\{1, \ldots, 2 k\}$ into $k$ pairs $\left(a_{1}, b_{1}\right), \ldots,\left(a_{k}, b_{k}\right)$ there exist permutations $P\left(a_{1}, b_{1}\right), \ldots, P\left(a_{k}, b_{k}\right)$ such that:

1. $l\left(P\left(a_{i}, b_{i}\right)\right)=a_{i}, r\left(P\left(a_{i}, b_{i}\right)\right)=b_{i}$ for each $i=1, \ldots, k$, and

2. For every $x, y \in A$ there are $i, j$ such that $x<y$ in $P\left(a_{i}, b_{i}\right)$ and $y<x$ in $P\left(a_{j}, b_{j}\right)$.

Proof. Let $P$ be an arbitrary permutation over the set $A \backslash\left\{a_{1}, a_{2}, b_{1}, b_{2}\right\}$, $\operatorname{Rev}(P)$ be obtained from $P$ by writing it in the reverse order, $P^{\prime}$ be an arbitrary permutation over the set $A \backslash\left\{a_{1}, a_{2}, a_{3}, b_{1}, b_{2}, b_{3}\right\}$ and for each $i=4, \ldots, k$ let $P_{i}$ be an arbitrary permutation over the set $A \backslash\left\{a_{i}, b_{i}\right\}$. Define the sought permutations as follows: $P\left(a_{1}, b_{1}\right)=$ 
$a_{1} b_{2} P a_{2} b_{1}, P\left(a_{2}, b_{2}\right)=a_{2} b_{1} \operatorname{Rev}(P) a_{1} b_{2}, P\left(a_{3}, b_{3}\right)=a_{3} b_{2} a_{1} P^{\prime} b_{1} a_{2} b_{3}$ and $P\left(a_{i}, b_{i}\right)=a_{i} P_{i} b_{i}$ for each $i=4, \ldots, k$. It is straightforward to verify that both requirements of the lemma hold for these permutations.

Note that for $n \in\{2,4\}$ Lemma 6 is not true.

Theorem 7. If $n \geq 5$ then the crown graph $H_{n, n}$ is $\lceil n / 2\rceil$-representable.

Proof. It is sufficient to prove the theorem only for $n=2 k, k \geq 3$, because the case of $n=2 k-1$ is obtained from the case of $n=$ $2 k$ by removing all occurrences of the letters $2 k$ and $(2 k)^{\prime}$ from the respective word. First, consider the following $k$-uniform word, where the permutations $P(x, y)$ s are defined in Lemma 6 and $P\left(x^{\prime}, y^{\prime}\right)$ s are obtained from these by adding primes.

$$
w^{\prime}=P(1,2) P\left(2^{\prime}, 3^{\prime}\right) P(3,4) P\left(4^{\prime}, 5^{\prime}\right) \cdots P(n-1, n) P\left(n^{\prime}, 1^{\prime}\right) .
$$

It follows from the property 2 in Lemma 6 that $w^{\prime}$ represents the complete bipartite graph $K_{n, n}$. Shift $w^{\prime}$ cyclicly one position to the left to obtain the word $w^{\prime \prime}$ where for every even $i$ there is exactly one occurrence of the factor $i i^{\prime}$ and for every odd $i$ there is exactly one occurrence of the factor $i^{\prime} i$. Let $w$ be the word obtained from $w^{\prime \prime}$ by switching $i$ and $i^{\prime}$ in each of these factors. This operation makes the subword induced by $i$ and $i^{\prime}$ non-alternating (thus removing the edges $i i^{\prime}$ in $K_{n, n}$ ) but does not affect any other alternations in the word. Therefore, $w k$-represents $H_{n, n}$, as desired.

Note that for $n<4$ the graph $H_{n, n}$ is 2-word-representable, which is given by the words $w_{1}=11^{\prime} 1^{\prime} 1, w_{2}=12^{\prime} 21^{\prime} 21^{\prime} 12^{\prime}$ and $w_{3}=$ $12^{\prime} 3^{\prime} 123^{\prime} 1^{\prime} 231^{\prime} 2^{\prime} 3$, respectively (see pages 172 and 173 in [8]). As for $n=4$, note that $H_{4,4}$ is the three-dimensional cube, which is the prism graph $\mathrm{Pr}_{4}$. Thus, $H_{4,4}$ is 3-word-representable by Proposition 15 in [9] and it is not 2-word-representable by Theorem 18 in [7]. An example of 3-representation of $H_{4,4}$ given on page 90 in [8] is

$414^{\prime} 343^{\prime} 231^{\prime} 12^{\prime} 24^{\prime} 1^{\prime} 3^{\prime} 44^{\prime} 2^{\prime} 33^{\prime} 11^{\prime} 22^{\prime}$.

\section{Concluding remarks}

In this paper we found the representation number of any crown graph $H_{n, n}$ solving at once two open problems in [8]. $H_{n, n}$ has the largest 
known representation number among bipartite graphs on $2 n$ vertices. We would like to propose the following conjecture that would be interesting to prove or disprove.

Conjecture 1. Each bipartite graph on $n$ vertices has representation number at most $n / 4$.

\section{References}

[1] R. Beigel, D. Eppstein. 3-coloring in time O(1.3289n). J. Algorithms 54 (2) (2005) 168-204.

[2] E. J. L. Bell. Word-graph theory. PhD thesis, Lancaster University, 2011.

[3] I. M. Bomze, M. Budinich, P. M. Pardalos, M. Pelillo. "The maximum clique problem", Handbook of Combinatorial Optimization, 4, Kluwer Academic Publishers (1999) 1-74.

[4] J. Črný. Coloring circle graphs. Electronic Notes in Discr. Math. 29 (2007) 457-461.

[5] R. Graham, N. Zang. Enumerating split-pair arrangements, J. Combin. Theory, Series A 115, Issue 2 (2008), 293-303.

[6] M. Halldórsson, S. Kitaev, A. Pyatkin. Semi-transitive orientations and word-representable graphs. Discr. Appl. Math. 201 (2016) 164-171.

[7] S. Kitaev. On graphs with representation number 3. J. Autom., Lang. and Combin. 18 (2013) 2, 97-112.

[8] S. Kitaev, V. Lozin. Words and graphs. Springer, 2015.

[9] S. Kitaev, A. Pyatkin. On representable graphs. J. Autom., Lang. and Combin. 13 (2008) 1, 45-54.

[10] S. Kitaev, S. Seif. Word problem of the Perkins semigroup via directed acyclic graphs. Order 25 (2008) 3, 177-194.

[11] M. Koebe. On a new class of intersection graphs. In Jaroslav Nešetřil and Miroslav Fiedler, editors, Fourth Czechoslovakian Symposium on Combinatorics, Graphs and Complexity (Prachatice, 1990), volume 51 of Ann. Discrete Math., pages 141-143. North-Holland, Amsterdam, 1992.

[12] L. Lovász. Perfect graphs. Selected Topics in Graph Theory, 2, London: Academic Press (1983) 55-87. 
[13] S. Seif. The Perkins Semigroup has Co-NP-complete termequivalence problem. Int. J. Alg. Comp. (IJAC) 15(2) (2005) $317-326$. 